\section{Reumatología pediátrica. Breve historia y situación de la especialidad en España}

\section{Introducción}

Aunque resulte paradójico este editorial aborda la situación actual de una especialidad, la Reumatología Pediátrica (RP), que oficialmente no existe en España. Esto es así porque en el año 2014 las especialidades pediátricas continúan sin ser reconocidas oficialmente en nuestro país. El hecho de que no exista reconocimiento legal implica que ni existen unos requisitos mínimos para ejercer adecuadamente la especialidad ni se dispone de un plan formativo oficial. Por tanto, cualquier profesional proveniente tanto del campo de la Pediatría como de la Reumatología, independientemente de su formación específica en RP, puede atender en España a niños con enfermedades reumáticas. Esta situación, sin duda legal, puede repercutir y repercute en la calidad de la atención prestada.

A pesar de ello no hay que perder de vista el largo camino recorrido por nuestra especialidad que ha llevado a su pleno desarrollo y reconocimiento en muchos países de la Unión Europea.

\section{La Reumatología Pediátrica como especialidad diferenciada}

La Reumatología Pediátrica inició su andadura como especialidad en los años cincuenta del siglo pasado tanto en EE.UU. (creación de los Special Treatment Centers for Rheumatic disorders) como en Europa (Taplow, Reino Unido). En aquellos años los niños eran considerados como "adultos pequeños" [1] y la Pediatría valorada por muchos internistas como una especialidad de segundo orden, por lo que los niños con enfermedades reumáticas eran atendidos por especialistas de adultos. Los primeros pediatras que empezaron a atender a niños con estas enfermedades carecían lógicamente de la formación ne-
Jaime de Inocencio Arocena ${ }^{1}$, Jordi Antón López $^{2}$

1 Unidad de Reumatología Pediátrica. Hospital Universitario 12 de Octubre, Madrid.

2 Unidad de Reumatología Pediátrica, Hospital Sant Joan de Déu, Esplugues, Barcelona.

\section{Correspondencia:}

”- jaime.inocencio@salud.madrid.org 
cesaria en reumatología, por lo que se formaron con reumatólogos (Dres. Joseph Levinson y Sydney Stillman entre otros en EE.UU., Prof. Eric Bywaters en Reino Unido). Muchos otros reumatólogos, sin embargo, abordaban tanto el hecho de enfermar como la propia enfermedad y su impacto en la familia con una perspectiva muy diferente de la de los pediatras, lo que ya en aquellos años produjo tensiones entre estos colectivos [2].

Durante las dos décadas siguientes los pediatras y reumatólogos que atendían a niños con patología reumática observaron que las artritis pediátricas, con un predominio de mono/oligoartritis y de poliartritis asimétricas, eran claramente diferentes de la artritis reumatoide del adulto, rechazando de plano el concepto de que los niños eran pequeños adultos. Este hecho indicaba la necesidad de que para tratar a niños con enfermedades reumáticas era necesario adquirir una formación específica en $\mathrm{RP}$, independientemente de la formación de origen de cada especialista.

La década de 1970 fue un periodo especialmente importante. La Sociedad Americana de Reumatología (entonces American Rheumatism Association, ARA) desarrolló la base de datos ARAMIS (American Rheumatism Association Medical Information System) que facilitaba la recogida sistemática de información clínica y de laboratorio de pacientes con enfermedades reumáticas. El Dr. Joseph Levinson, miembro del Subcomité de criterios de clasificación de la Artritis Reumatoide Juvenil de la ARA, completó pacientemente la información relativa a la presentación y curso clínico de centenares de pacientes pediátricos seguidos por él en la Universidad de Cincinnati [3] lo que facilitó la publicación de los primeros criterios de clasificación de la denominada Artritis Reumatoide Juvenil [4] y posteriormente su revisión [5]. Estudios publicados una década más tarde destacaron la utilidad de la clasificación, así como la necesidad de recoger no sólo información de la forma de comienzo de la enfermedad sino también del curso clínico de la misma a los 6 meses del inicio [6].

Uno de los pioneros en el campo de la Reumatología Pediátrica, el Dr. Earl Brewer, publicó una magnífica revisión del nacimiento de la especialidad $[2,7,8]$. Él presidió tanto el Subcomité de criterios de clasificación de la Artritis Reumatoide Juvenil como el Pediatric Rheumatology Collaborative Study Group (PRCSG) estadounidense, creado en 1973 con el objeto de coordinar ensayos clínicos en RP; asimismo, Earl Brewer fue uno de los principales impulsores de la primera reunión de reumatólogos pediátricos, la "ARA Conference on Rheumatic Diseases of Childhood". Esta conferencia se celebró en Marzo de 1976 en Park City, Utah, EE.UU. [9], lo que representó el primer congreso de Reumatología Pediátrica celebrado en el mundo. Esta reunión supuso el inicio de la consolidación de la especialidad [10], que concluyó formalmente en EE.UU. en 1992 al ser reconocida oficialmente mediante la convocatoria por el American Board of Pediatrics del primer examen de acreditación en RP, el Subboard of Pediatric Rheumatology.

En Europa la especialidad siguió unos derroteros similares. En 1947 el Dr. Eric Bywaters fue nombrado director de una nueva Unidad Especial para el Reumatismo Juvenil en el Canadian Red Cross Memorial Hospital de Taplow, Reino Unido [11]. El hospital nació con la finalidad de estudiar el reumatismo infantil, representado en aquella época por la fiebre reumática. Muy pronto se incorporaría al equipo médico una joven pediatra que en poco tiempo se convertiría en un referente internacional, la Dra. Barbara Ansell [12]. Al declinar la fiebre reumática el objetivo de estudio del hospital se centró, afortunadamente para la especialidad, en las artritis crónicas pediátricas, lo que hizo que Taplow se mantuviera durante muchos años como un referente en la formación de especialistas en RP. Miembros muy destacados de la 
reumatología pediátrica norteamericana, como los Dres. James Cassidy, Jane Schaller, Virgil Hanson o Chester Fink, hicieron estancias en Taplow.

El propio Dr. Bywaters hizo mención en la reunión de Park City de 1976 al nacimiento de la especialidad con una frase que ha pasado a los anales de la RP: "I think I saw it arrive, although I cannot specify its birthday or place and I am damned if I can read the father's signature on the birth certificate" [Creo que la vi llegar aunque no puedo precisar ni la fecha ni el lugar de nacimiento, y que me aspen si puedo leer la firma del padre en el certificado de nacimiento].

Ambos extremos del Atlántico iniciaron una fructífera relación en 1976 con la colaboración EE.UU.-URSS [7] que condujo a la realización del primer ensayo clínico aleatorizado, doble ciego y controlado con placebo de metotrexato en el tratamiento de la Artritis Reumatoide Juvenil [13], que marcó un antes y un después en la terapia de la enfermedad. Ese mismo año figuras tan significativas de la RP europea como el Dr. Eric Bywaters, la Dra. Barbara Ansell o el Dr. Philip Wood asistieron a la reunión de Park City y en 1977 se organizó un congreso internacional en Oslo, Noruega, con una nutrida participación de colegas europeos y americanos. Ese mismo año se publicaron los criterios de clasificación de la Artritis Crónica Juvenil [14].

En lo referente al reconocimiento de la RP como especialidad en Europa, la formación especializada en RP fue aprobada en Reino Unido en 1994 por el Royal College of Physicians y en 1996 por el Royal College of Paediatrics and Child Health. Otros países han seguido el ejemplo de Reino Unido y reconocido oficialmente la especialidad.

En 1996 nació el equivalente europeo del PRCSG norteamericano, el Pediatric Rheumatology International Trials Organization (PRINTO), con el impulso personal del Dr. Alberto Martini, entonces en Pavía, (Italia) y la colaboración del Dr. Nicola Ruperto como asesor científico, que tanto ha contribuido al desarrollo de nuestra especialidad a través del diseño y coordinación de múltiples estudios multicéntricos llevados a cabo en Europa, México, Sudamérica y Oceanía.

En 1996 se publicaron, asimismo, los criterios de clasificación de la Artritis Idiopática Juvenil (AIJ) propuestos por el Comité Pediátrico de ILAR (International League of Associations for Rheumatology) con el apoyo de la Organización Mundial de la Salud (OMS) [15]. Estos criterios y la nueva denominación de las artritis inflamatorias pediátricas pretendían unificar el diagnóstico y la nomenclatura de la enfermedad a ambos lados del Atlántico. El comité se creó durante el XVIII Congreso de la ILAR, celebrado en Barcelona en 1993. Los primeros criterios de AIJ se redactaron en la reunión de ILAR de Santiago de Chile de 1994, siendo posteriormente revisados en dos ocasiones, en 1997 en Durban, República de Sudáfrica [16], y en 2001 en Edmonton, Canadá [17], antes de ser aceptados y utilizados internacionalmente. Estos criterios culminaban el proceso iniciado en los años 1950 de alcanzar un consenso en la clasificación la enfermedad [18].

Finalmente, en 1997 se creó la Sociedad Europea de Reumatología Pediátrica (Paediatric Rheumatology European Society, PReS) fruto de la coordinación de esfuerzos de los líderes europeos de entonces: la Dra. Patricia Woo (Londres), que fue su primer presidente, la Dra. Anne Marie Prieur (París), el Dr. Alberto Martini (Pavía), presidente en la actualidad, y el Dr. Wietse Kuis (Utrecht). Desde su creación PReS ha organizado anualmente los congresos europeos de RP con la particularidad de que cada 3 años se celebran de manera conjunta con los congresos de la European League Against Rheumatism (EULAR). 


\section{Inicios de la Reumatología Pediátrica en España}

La formalización de la especialidad en España comenzó en 1992. Ese año un grupo de pediatras (Dres. Inmaculada Calvo, Valencia; Julia GarcíaConsuegra, Madrid; Javier Gómez-Reino, Madrid, y Enrique González Pascual, Barcelona) y de reumatólogos (Dres. Cristina Arnal, Barcelona; Luis Carreño, Madrid; Mari Luz Gámir, Madrid; Javier López Longo, Madrid, e Isabel Rotés, Barcelona) que venían trabajando en este campo crearon el Grupo Español de Reumatología Pediátrica (GERP) con la intención de dar una estructura organizativa a una situación que ya existía de hecho [19]. Este Grupo organizó varias Jornadas de Actualización en Reumatología Pediátrica.

En lo referente al reconocimiento de la especialidad por las sociedades científicas nacionales este tuvo lugar en 1995 cuando el Grupo de Trabajo de Reumatología Pediátrica de la Asociación Española de Pediatría (AEP), constituido años antes por un grupo de pediatras, se convirtió en la nueva Sección de Reumatología Pediátrica de la AEP.

En 1998 se firmó el acta fundacional de la Sociedad Española de Reumatología Pediátrica (SERPE), albergando en su seno a todos aquellos pediatras, reumatólogos y personal sanitario y no sanitario interesado en la RP. Un año antes, 1997, tuvo lugar el I Congreso Nacional de Reumatología Pediátrica en Valencia, celebrándose hasta la fecha estos congresos con carácter bienal; el último, el $10^{\circ}$, tuvo lugar en 2013 en Granada.

Desde 2004 se organizan reuniones de actualización, los denominados Foros de la SERPE, los años en que no se celebra el Congreso Nacional. El primero tuvo lugar en Barcelona, estando programado el correspondiente a 2014 en la ciudad de Vigo.
En el año 2010 la SERPE organizó en Valencia el $17^{\circ}$ Congreso de la Sociedad Europea de Reumatología Pediátrica. Este congreso supuso el reconocimiento de la Sociedad Europea de Reumatología Pediátrica (PReS) a la labor desarrollada por la SERPE y fue un gran éxito tanto por el número de asistentes llegados de todo el mundo como por el alto nivel científico de las ponencias y comunicaciones presentadas. Este congreso marcó la mayoría de edad de la especialidad en nuestro país.

\section{Formación en Reumatología Pediátrica}

Después de repasar los orígenes de la especialidad puede parecer redundante insistir en la necesidad de tener una adecuada formación específica. Sin embargo, parece que en España se está volviendo a la situación que existía antes de los años 1990 en Europa y Norteamérica, impulsando la creencia errónea de que cualquier especialista, sea pediatra o reumatólogo, puede atender adecuadamente a estos niños por el mero hecho de haber recibido una mínima formación durante su residencia, haber realizado alguna breve rotación en RP, o incluso por haberse inscrito a algún curso teórico presencial u online sobre la materia. Si esta fuera la propuesta formativa de otras especialidades pediátricas, como la cardiología o la oncología, no dejaría indiferente a nadie.

Esta situación no es exclusiva de España y motivó un editorial de la revista Pediatric Rheumatology Online Journal en 2007, en donde se abordaba la falta de idoneidad para el ejercicio de la especialidad tanto de pediatras como de reumatólogos sin formación específica en RP [20].

El Comité de Educación de PReS desarrolló un programa de formación en reumatología pediátrica que recogía explícitamente las necesidades formativas tanto teóricas como prácticas necesarias para ejercer adecuadamente la especialidad. Este programa fue 
aceptado por la Sociedad Europea de Reumatología (European League Against Rheumatism, EULAR) y, posteriormente, tanto por la European Academy of Paediatrics como por el European Board of Paediatrics en la reunión mantenida en Grado, Italia, el 27 de abril de 2008 [21]. Uno de los requisitos exigidos para la acreditación de la especialidad por el European Board of Paediatrics era tener una sólida formación en Pediatría dado que, siguiendo al Prof. Maximilian Zach, presidente en 2009 de la European Academy of Paediatrics/Union of European Medical Specialists (UEMS) Section of Paediatrics, "we always treat sick children and not just disordered organ systems" [siempre tratamos a niños enfermos y no simplemente órganos alterados] [22].

\section{Situación de la Reumatología Pediátrica en España}

Hasta la fecha el mejor análisis de la situación de la especialización pediátrica en España comparada con la de otros países Europeos es el realizado por la Asociación Española de Pediatría en el Libro Blanco de las Especialidades Pediátricas publicado en 2011 [23].

La SERPE intentó realizar en 2012 y 2013 un mapa de la actividad asistencial, dependencia jerárquica, etc. de los distintos servicios hospitalarios que prestan atención a niños con enfermedades reumáticas en España. Para ello se utilizó el mismo formato de encuesta usado en el Libro Blanco. Lamentablemente, la iniciativa contó con un muy escaso seguimiento, por lo que en la actualidad es imposible dar cifras precisas del número de facultativos, de su formación o del número de niños atendidos en las distintas Unidades de Reumatología Pediátrica del país.

Dado que el número de profesionales que trabajan en este campo es limitado, sí son conocidas aunque no cuantificables las grandes diferencias existentes entre las distintas Comunidades Autónomas e incluso entre los distintos centros dentro de una misma Comunidad.

En la actualidad la formación de los profesionales en RP es muy heterogénea, con una mayoría de especialistas cuya formación específica ha consistido en rotaciones inferiores a periodos de 3-6 meses por unidades de referencia o no de RP. No es infrecuente, por tanto, la existencia de facultativos "autotitulados", esto es, aquellos que por motivos organizativos o asistenciales y con una limitada preparación específica han tenido que hacerse cargo de la atención a niños con este tipo de enfermedades. Como consecuencia, la formación en técnicas básicas en reumatología como las artrocentesis, infiltraciones, ecografía músculo-esquelética, etc. es ampliamente variable, con centros que directamente no las realizan o que deben contar con la ayuda de otros servicios (Traumatología, Rehabilitación, Radiología, etc.) para acceder a las mismas.

En lo referente al modelo de atención asistencial, el más habitual es a tiempo parcial, con una frecuencia de consultas que oscilan entre 2 a la semana a 2 al mes, contándose prácticamente con los dedos de una mano los centros cuyos profesionales se dedican de manera exclusiva a la RP. La mayoría de los profesionales alterna la atención a niños con enfermedades reumáticas con consultas de pediatría general, enfermedades infecciosas pediátricas, etc., o bien de Reumatología general, lo que señala que sólo dedican entre un diez y, en el mejor de los casos, un cuarenta por ciento de su tiempo a la RP.

Uno de los requisitos exigidos por PReS para poder acreditar un programa de formación en RP es que éste disponga de al menos 2 facultativos a tiempo completo [24]. En todo el país sólo hay 3 centros con al menos dos agendas de consulta abierta de 
manera diaria, el Hospital Sant Joan de Déu (Barcelona), el Hospital La Paz (Madrid) y el Hospital La Fe (Valencia).

Recientemente, 8 de Agosto de 2014, se ha publicado en el Boletín Oficial del Estado el Real Decreto 639/2014 que regula la troncalidad, la reespecialización troncal y las áreas de capacitación específica [25]. Este decreto abre la puerta al proceso de reconocimiento oficial de las especialidades pediátricas, entre ellas la RP, y brinda soluciones como la creación de programas de formación por el sistema de residencia o la acreditación de Unidades Docentes, permitiendo trazar un camino que nos equipare a Estados Unidos, Canadá u otros países europeos.

\section{Conclusión}

La reumatología pediátrica es una especialidad que a pesar de su juventud ha recorrido un largo camino hasta su consolidación. En la actualidad es una especialidad reconocida en muchos países que tiene claramente definido: (1) qué patologías tanto inflamatorias como no inflamatorias son de su competencia; (2) qué formación teórico-práctica debe tener los profesionales que la ejercen; y (3) qué criterios deben cumplir las Unidades donde se lleva a cabo la asistencia.

A la vista de todo lo anterior no deja de resultar un tanto frustrante comprobar el panorama actual de la atención a niños con enfermedades reumáticas en España, en donde cualquier pediatra o reumatólogo que lo desee o a quienes sus respectivos responsables jerárquicos designen puede hacerse cargo de la atención a estos pacientes de manera más o menos ocasional y con una variable formación específica. Esta situación era la habitual cuando comenzó el desarrollo de las especialidades pediátricas en los años setenta del siglo pasado, pero está muy lejos de ser el modelo idóneo cuarenta años después, en 2014. Nuestros pacientes merecen algo mejor.

\section{Referencias}

1. Schaller, JG. The history of pediatric rheumatology. Peditr Res 2005; 58: 997-1007. [Consultado el 21 de Septiembre de 2014]. Disponible en http://www.nature.com/pr/journal/v58/n5/pdf/ pr2005810a.pdf

2. Brewer EJ. A peripatetic pediatrician's journey into pediatric rheumatology. Pediatr Rheumatol Online J. 2007 May 23;5:11. [Consultado el 16 de Agosto de 2014]. Disponible en http:// www.ped-rheum.com/content/pdf/1546-0096-5-11.pdf

3. Wolfe F. A short history of data banking in the United States from 1974 to 2003. J Rheumatol 2004;31 (Suppl 69):41-5. [Consultado el 21 de Septiembre de 2014]. Disponible en http:// www.jrheum.com/subscribers/04/69/41.html

4. Brewer, EJ. Jr, Bass, JC., Cassidy, JT., Duran, BS., Fink, CW., Jacobs, JC. et al. Criteria for classification of Juvenile Rheumatoid Arthritis. Bull Rheum. Dis 1972; 23: 712-9.

5. Brewer, EJ. Jr, Bass, JC., Baum, J., Cassidy, JT., Fink, CW., Jacobs, JC. et al. Current proposed revision of JRA criteria. Arthritis Rheum 1977; 20 (Suppl. 2): 195-9.

6. Cassidy, JT., Levinson, JE., Bass, JC., Baum, J., Brewer, EJ. Jr, Fink, CW. et al. A study of classification criteria for a diagnosis of Juvenile Rheumatoid Arthritis. Arthritis Rheum 1986; 29: 27481.

7. Brewer EJ. A peripatetic pediatrician's journey into pediatric rheumatology: Part II. Pediatr Rheumatol Online J. 2007 Jun 21;5:14. [Consultado el 9 de Marzo de 2014]. Disponible en http://www.ped-rheum.com/content/pdf/1546-0096-5-14.pdf.

8. Brewer EJ. A peripatetic pediatrician's journey into pediatric rheumatology: Part III. Pediatr Rheumatol Online J. 2007 Sep 25;5:17. [Consultado el 9 de Agosto de 2014]. Disponible en http://www.ped-rheum.com/content/pdf/1546-0096-5-17.pdf.

9. Proceedings of the First ARA Conference on the Rheumatic Diseases of Childhood. Park City, Utah, March 22-25, 1976. Arthritis Rheum. 1977; 20 (2 Suppl.): 145-628.

10. Woo P, Petty R. Looking at paediatric rheumatology over the past 50 years. Rheumatology 2011;50:1533-6. [Consultado el 21 de Septiembre de 2014]. Disponible en http://rheumatology. oxfordjournals.org/content/50/9/1533.full.

11. Ansell, BM. Taplow reminescences. J Rheumatol. 1992; 19 (Suppl. 33): 105-7.

12. Schaller, JG. Tribute to Barbara Ansell, 1923-2001. J Rheumatol. 2002; 29: 2237-8. 
13. Giannini EH, Brewer EJ, Kuzmina N, Shaikov A, Maximov A, Vorontsov I et al. Methotrexate in resistant juvenile rheumatoid arthritis. Results of the USA-USSR double-blind, placebocontrolled trial. New Engl J Med 1992;326:1043-9. [Consultado el 21 de Septiembre de 2014]. Disponible en http://www.nejm. org/doi/pdf/10.1056/NEJM199204163261602

14. European League Against Rheumatism EULAR Bulletin No. 4 : Nomenclature and classification of arthritis in children. Basel: National Zeitung AG; 1977.

15. Fink, CW. Proposal for the development of classification criteria for idiopathic arthritides of childhood. J Rheumatol. 1995; 22: 1566-9.

16. Petty, RE., Southwood, TR., Baum, J., Bhettay, E., Glass, DN., Manners, P. et al. Revision of the proposed classification criteria for Juvenile Idiopathic Arthritis: Durban, 1997. J Rheumatol. 1998; 25: 1991-4.

17. Petty, RE., Southwood, TR., Manners, P., Baum, J., Glass, DN., Goldenberg, J. et al. International league of associations for rheumatology classification of Juvenile Idiopathic Arthritis: second revision, Edmonton, 2001. J Rheumatol. 2004; 31: 390-2.

18. Rauz S, Murray PI, Southwood TR. Juvenile idiopathic arthritis and uveitis: the classification conundrum. Eye 2000;14:81720. [Consultado el 21 de Septiembre de 2014]. Disponible en http://www.nature.com/eye/journal/v14/n6/pdf/eye2000229a. pdf?origin=publication_detail

19. Grupo Español de Reumatología Pediátrica. Registro nacional de enfermedades reumáticas infantiles. An Esp Pediatr. 1996; 45 : 346-50.

20. Spencer, $\mathrm{CH}$. Why should pediatric rheumatology be recognized as a separate subspecialty: An open letter to medical councils and government agencies. Pediatr Rheumatol. 2007; 21 (5): 21.

21. Paediatric Rheumatology European Society (PReS) Education Committee. European syllabus and training programme in Paediatric Rheumatology. Revision 2008 [Consultado el 11 de Agosto de 2014]. Disponible en http://www. pres.org.uk/index.php?option $=c o m \_d o c m a n \& \operatorname{task}=d o c_{-}$ view\&gid=35\&ltemid $=26$.

22. Asociación Española de Pediatría. Libro Blanco de las Especialidades Pediátricas. Anexo 3. Carta del presidente de la European Academy of Paediatrics/Union of European Medical Specialists (UEMS) Section of Paediatrics al presidente del European Board of Rheumatology. Anexo E14-6, páginas 3702. [Consultado el 16 de Agosto de 2014]. Disponible en http:// www.aeped.es/sites/default/files/documentos/anexos_03.pdf.

23. Asociación Española de Pediatría. Libro Blanco de las Especialidades Pediátricas. [Consultado el 16 de Agosto de 2014]. Disponible en http://www.aeped.es/documentos/libroblanco-las-especialidades-pediatricas.
24. Asociación Española de Pediatría. Libro Blanco de las Especialidades Pediátricas. Anexo 3. Anexo E14-2: Minimum criteria for Paediatric Rheumatology training services, Paediatric Rheumatology European Society; Anexo E14-3 a E14-5: Training centre acreditation and visitation program. Paediatric section of UEMS (Union of European Medical Specialists). Páginas 35369. [Consultado el 16 de Agosto de 2014]. Disponible en http:// www.aeped.es/sites/default/files/documentos/anexos_03.pdf.

25. España. Real Decreto 639/2014, de 25 de Julio, por el que se regula la troncalidad, la reespecialización troncal y las áreas de capacitación específica, se establecen las normas aplicables a las pruebas anuales de acceso a plazas de formación y otros aspectos del sistema de formación sanitaria especializada en Ciencias de la Salud y se crean y modifican determinados títulos de especialista. Boletín Oficial del Estado, 6 de agosto de 2014, núm 190, pp 63130-63167. [Consultado el 31 de Agosto de 2014]. Disponible en http://www.boe.es/boe/dias/2014/08/06/ pdfs/BOE-A-2014-8497.pdf.

\section{Opina sobre este artículo:}

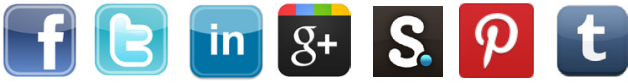

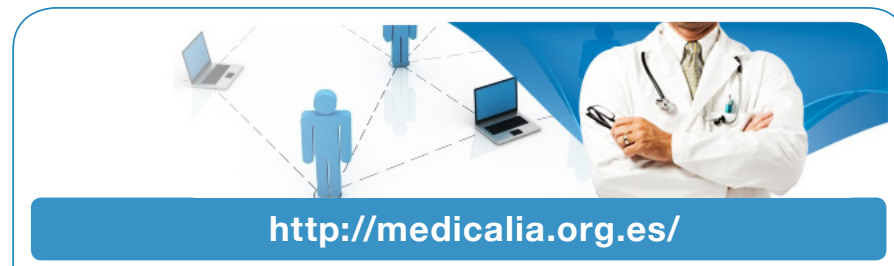

Los médicos disponen de una red social para intercambiar experiencias clínicas, comentar casos y compartir conocimiento. También proporciona acceso gratuíto a numerosas publicaciones. ¡Únase ahora!

\section{Publish with iMedPub}

\section{http://www.imedpub.com}

Acta Rheumatologica es una revista que tiene por fin la difusión de estudios clinicos relacionados con aspectos prácticos del diagnóstico, tratamiento y seguimiento de pacientes con patologia reumatológica, de estudios epidemiológicos relacionados con patologia inflamatoria y musculoesqueletica de presentacion común o infrecuente en la practica clinica tanto en población adulta como pediátrica, de casos clinicos de patologia poco habitual o de presentaciones inhabituales de patologia frecuente, de imágenes didácticas e ilustrativas en reumatologia y del estado actual e innovacion en la formacion especializada en reumatologia. 